\title{
Quercetin Relieves D-Amphetamine induced Manic-like Behavior through Activating TREK-1 Potassium Channels in Mice
}

\author{
Keke Ren ${ }^{1}$, Baolin Guo ${ }^{1}$, Rui $\mathrm{Li}^{1}$, Honghui Mao ${ }^{1}$, Han Yao ${ }^{1}$, Shenxi Wu${ }^{1}$, Zhantao Bai ${ }^{2}$, \\ and Wenting Wang ${ }^{1}$ \\ ${ }^{1}$ Fourth Military Medical University \\ ${ }^{2}$ Yan'an University
}

June 15,2020

\begin{abstract}
Background and Purpose: Quercetin is a prominent neuroprotective compound from flavonoids. Previous studies found it may relieve psychiatric disorders, cognition deficits, and memory dysfunction through anti-oxidation and/or radical scavenging mechanisms. In addition, Quercetin also was found to modulate the physiological function of a few types of ion channels. However, the detailed ionic mechanisms of quercetin's bioeffect remain unknown. Experimental Approach: We examined the effect neuronal activities changes in prefrontal cortex (PFC) and its ionic mechanisms upon quercetin application by using GCaMP calcium imaging and patch clamp in acute brain slices. Then we explored the potential ionic mechanism of quercetin on D-amphetamine induced manic-like using c-fos staining and the open field behavior test. Key Results: Quercetin reduced calcium influx triggered by PFC pyramidal neuronal activity. This effect was caused by increasing the rheobase of neuronal firing through decreasing membrane resistance upon quercetin treatment. Spadin, a TREK-1 potassium channel (a two-poredomain background potassium channel) inhibitor, could block the effect of quercetin on the membrane resistance and neuronal firing. And also, Spadin can block the neural protective effects of quercetin. In addtion, intraperitoneal injection of quercetin could relieve the manic hyperlocomotion of the mice induced by D-amphetamine, which can be partially alleviated by Spadin. Conclusion and Implications: Our results demonstrated that TREK-1 channel is a novel target on quercetin treatment, which could contribute to both the neuroprotection and anti-manic-like effects.
\end{abstract}

\section{Hosted file}

Quercetin-Keke manuscript@2020613.doc available at https://authorea.com/users/333571/ articles/459738-quercetin-relieves-d-amphetamine-induced-manic-like-behavior-throughactivating-trek-1-potassium-channels-in-mice 
A
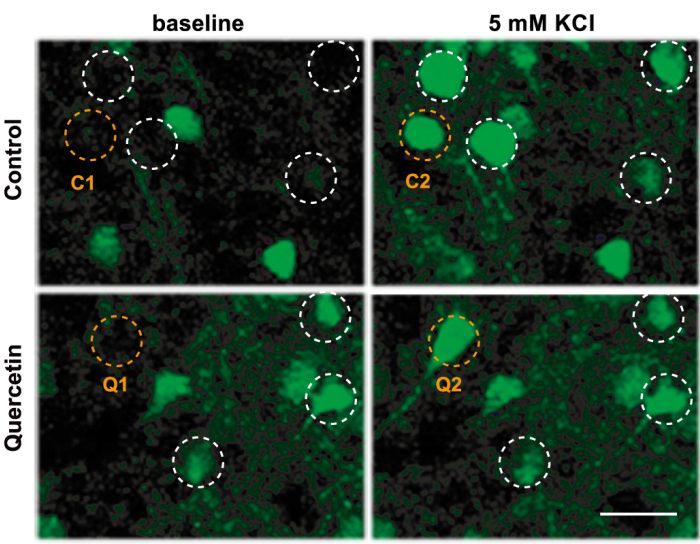

B

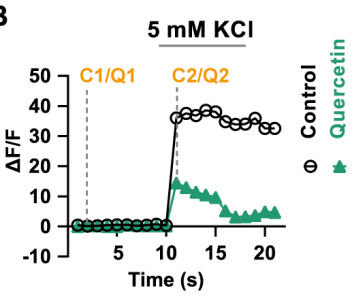

C

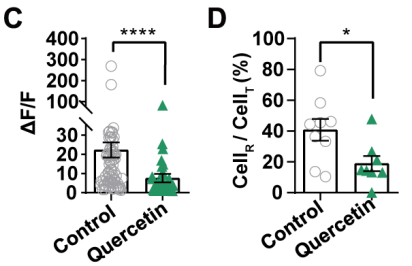

A

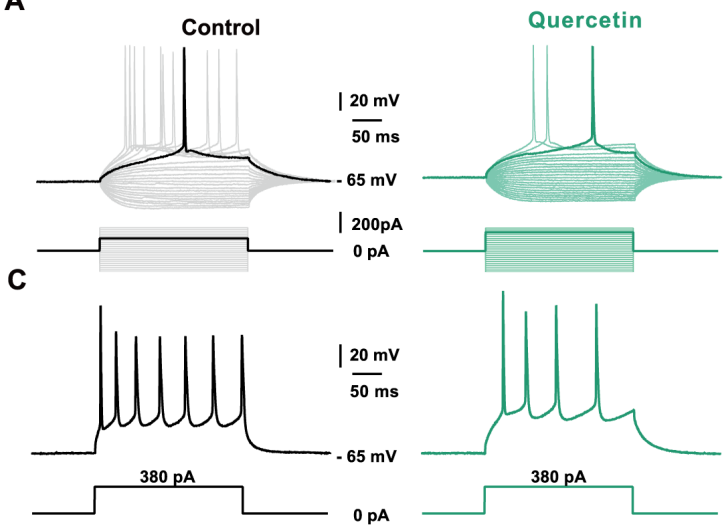

E

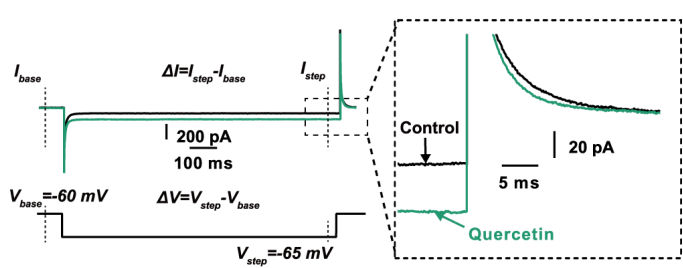

B

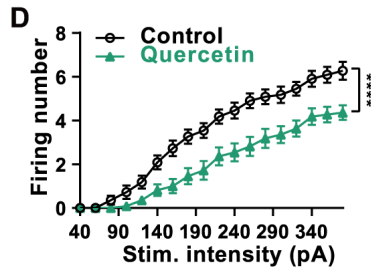

F

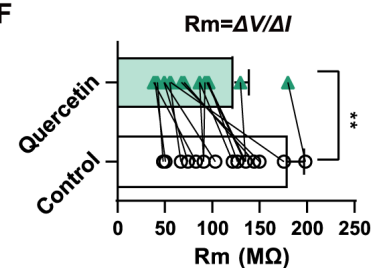

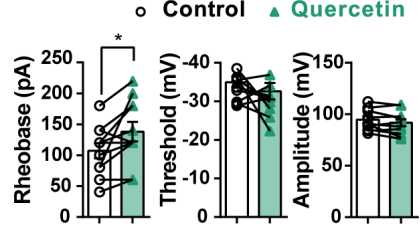


A

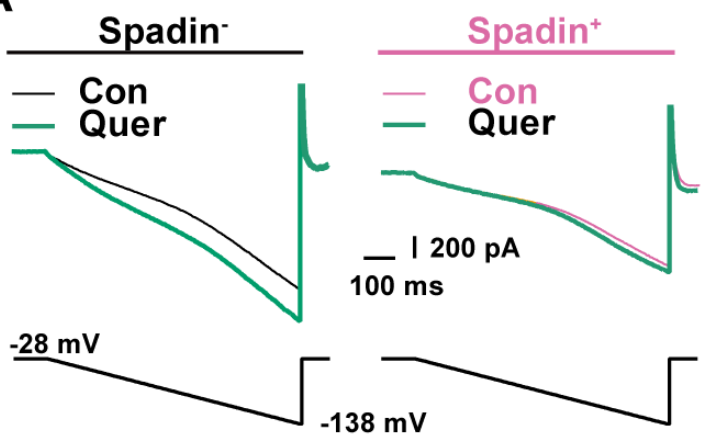

B

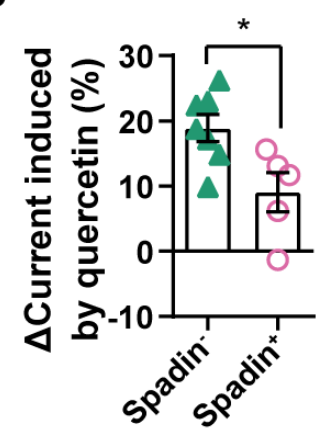

C

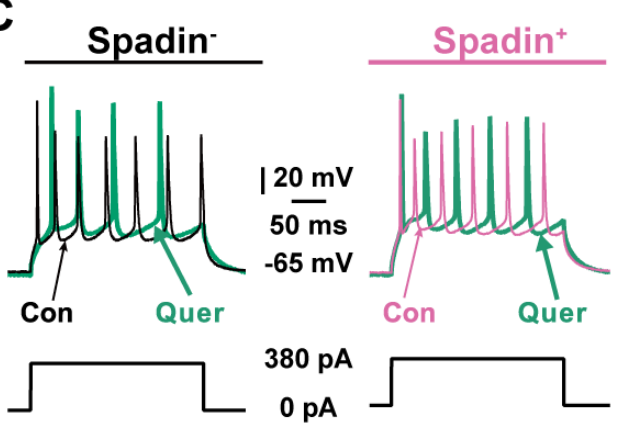

D

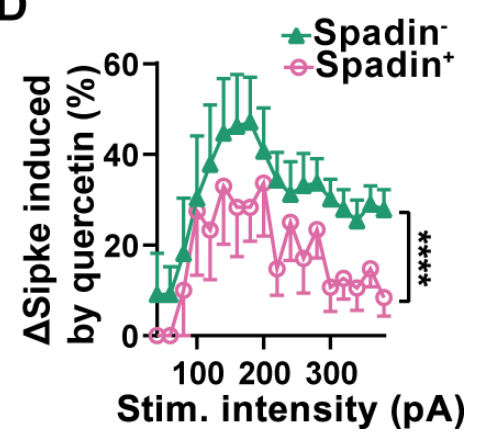

A

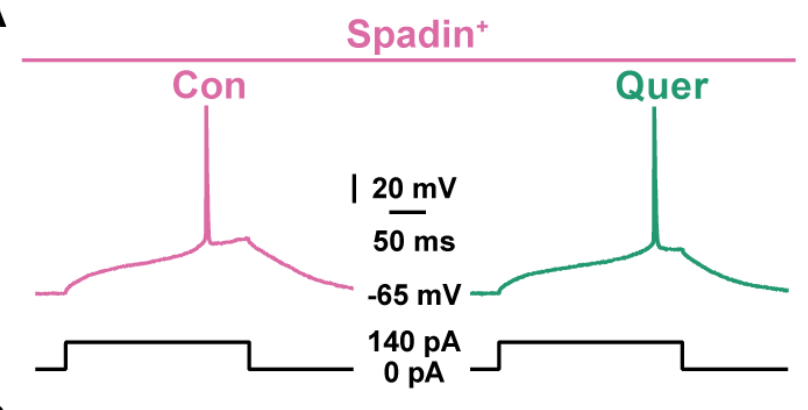

C

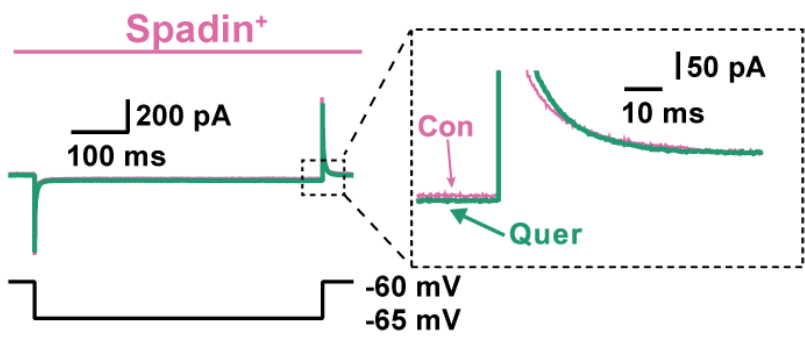

B

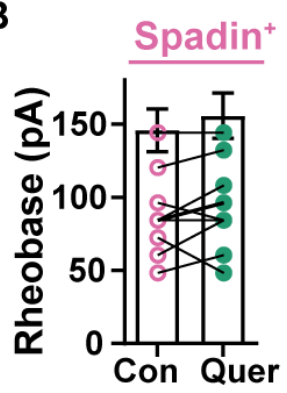

D

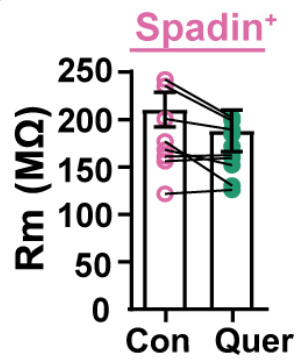


A
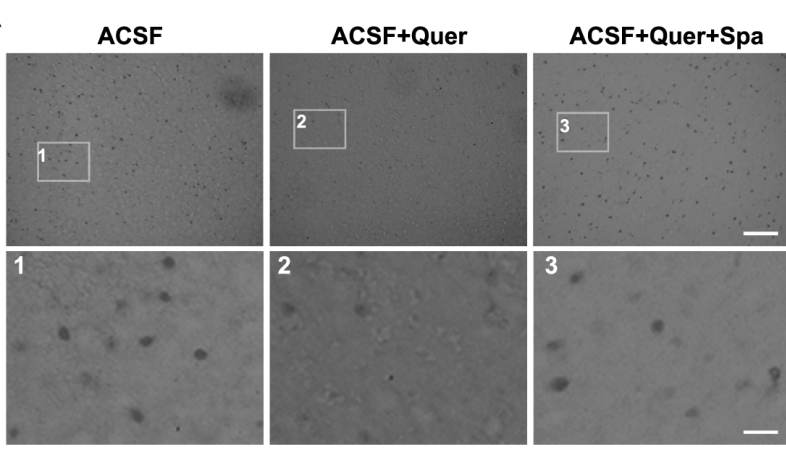

A

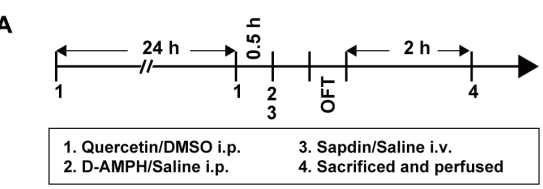

B
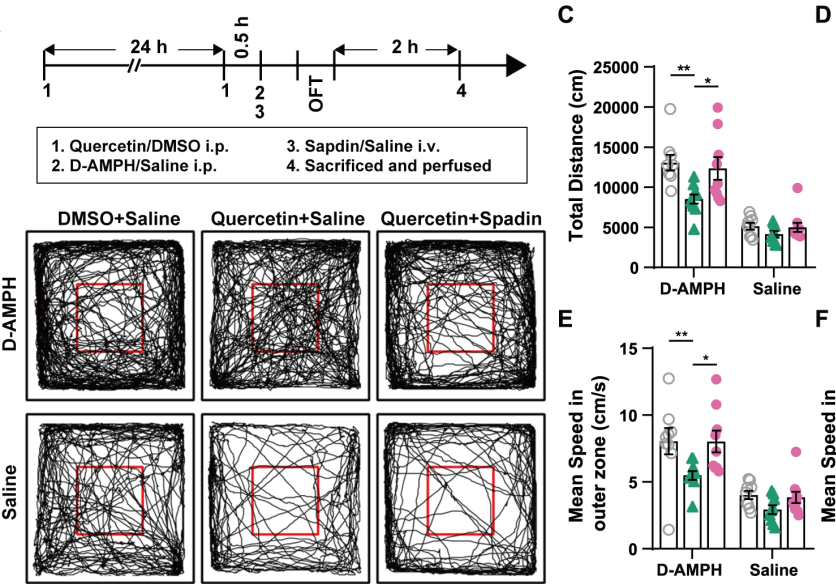
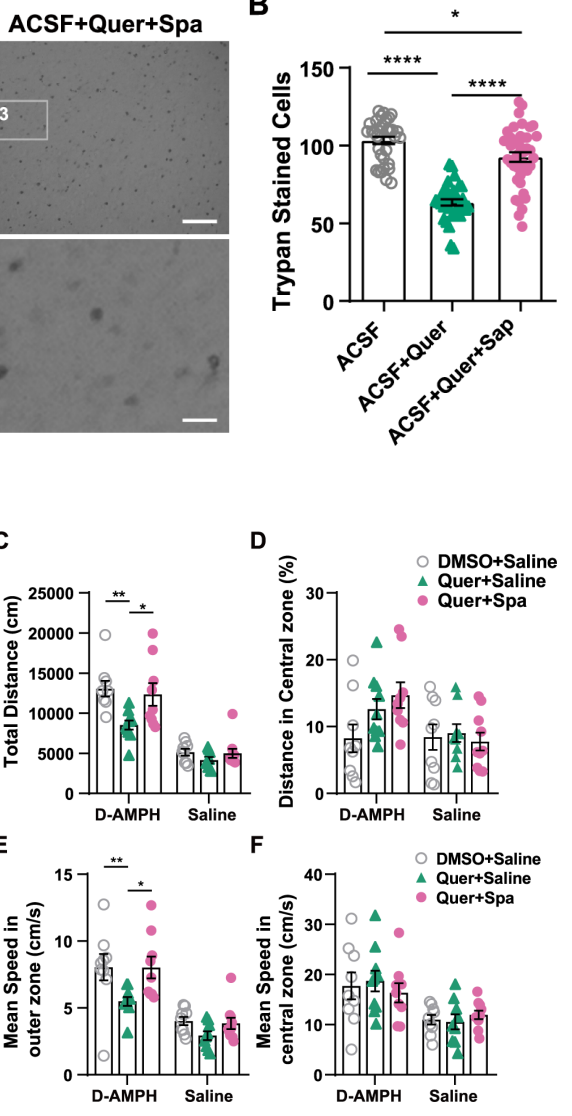

Ð $\quad$ DMSO+Saline uer+Spa

^
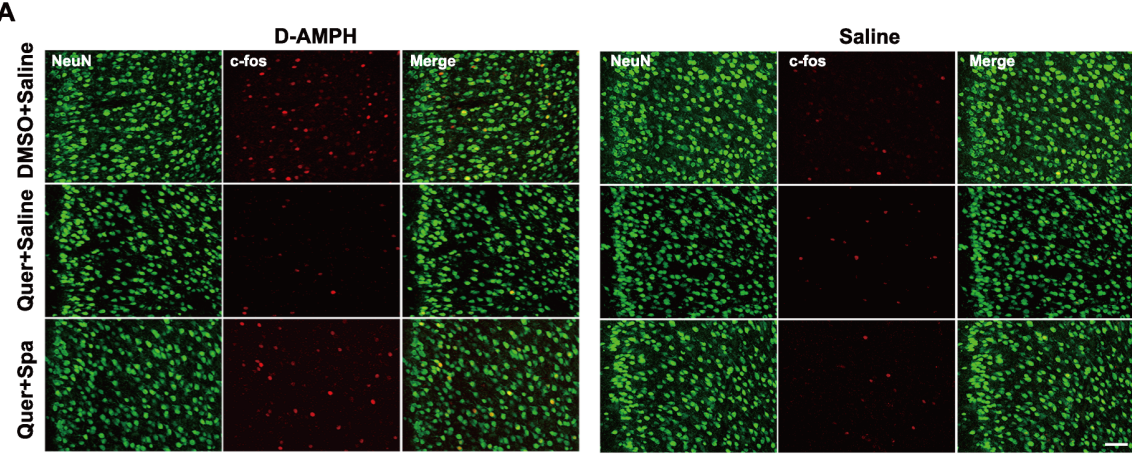

B
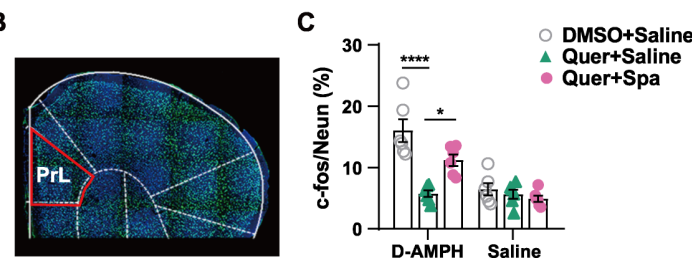